\title{
Lupine as a potential agent against diet-induced obesity through adenosine monophosphate- activated protein kinase pathway: crude oil versus nanoemulsion formulations
}

\author{
Omar Mahmoud Mohafez ${ }^{\oplus 1,3, *}$, Ibrahim Abdelrahman Alhaider², \\ Tamer Mohamed Shehata ${ }^{2,4}$, Maged Elsayed Mohamed ${ }^{2,5}$ \\ ${ }^{1}$ Department of Biomedical Sciences, College of Clinical Pharmacy, University of King Faisal, \\ Ahsaa, Kingdom of Saudi Arabia, ${ }^{2}$ Department of Pharmaceutical Sciences, College of Clinical \\ Pharmacy, University of King Faisal, Ahsaa, Kingdom of Saudi Arabia, ${ }^{3}$ Department of \\ Biochemistry, Faculty of Pharmacy, University of Al-Azhar, Assiut Branch, Egypt, ${ }^{4}$ Department of \\ Pharmaceutics and Industrial Pharmacy, Faculty of Pharmacy, University of Zagazig, Zagazig, \\ Egypt, ${ }^{5}$ Department of Pharmacognosy, Faculty of Pharmacy, University of Zagazig, Zagazig, Egypt
}

\begin{abstract}
Obesity represents a major challenge to the pharmaceutical community due to the minimal availability of anti-obesity drugs and the drawbacks of current weight-loss agents. The study described herein presents lupine oil, in two pharmaceutical formulations, as a potential anti-obesity agent via its effect on different physiological, biochemical, and hormonal parameters. Rats were divided into two groups; one group was continued on a standard commercial rodent diet and served as the non-obese control. The other group was fed a high-fat diet for 7 weeks to prepare an obese rat model. Then, the obese rats were divided into groups to receive $100 \mathrm{mg} / \mathrm{kg}$ of the crude lupine oil or nanoemulsion for 10 or 20 days. Lupine oil showed a potent body weight-reducing effect and improved insulin resistance. The oil altered obesity-induced hyperlipidemia and it enhanced the leptin/adiponectin/AMPK hormonal system in epididymal fat, serum, and liver, to which all the above physiological activities could be attributed. The nanoemulsion formulation of lupine oil significantly amplified the activity for all the above physiological and hormonal parameters when compared to the crude oil formulation. Lupine oil nanoemulsion could be used as a potential drug against diet-induced obesity.
\end{abstract}

Keywords: Adiponectin. Cholesterol. HDL. Leptin. Lupinus albus.

\section{INTRODUCTION}

Obesity is a major health and social problem with huge impacts on one's quality of life. The prevalence of obesity is linked with a disturbing increase in several diseases, such as diabetes mellitus, metabolic syndrome, chronic kidney disease, and many lifethreatening cardiovascular disorders (Sbihi et al., 2013). The number of obese individuals is increasing,

*Correspondence: O. Mohafez, Department of Biomedical Sciences, College of Clinical Pharmacy, University of King Faisal, P.P. 380, Ahsaa 31982, Kingdom of Saudi Arabia. Tel. +966549659590. E-mail: omarmohafez@azhar.edu.eg and millions of potential patients have been identified worldwide (Park, Usher, Foster, 2011). Adipokines, a group of hormones principally secreted from fat tissues, to modulate insulin sensitivity and fat metabolism (Rabe et al., 2008); they are thought to play essential roles in obesity and its related syndromes (Chang et al., 2010). Among the adipokines, leptin and adiponectin are considered the major players in obesity metabolism, as they primarily regulate adenosine monophosphate (AMP)-activated protein kinase (AMPK) activity (Dzamko, Steinberg, 2009). Leptin activates many kinases and signal transducers, which inhibit orexigenic peptides and activate anorexigenic 
peptides (Ahima, 2006). Leptin restricts food intake and suppresses hypothalamic AMPK activity, while adiponectin stimulates hypothalamic AMPK, which controls energy expenditure (Stark, Ashley, Andrews, 2013). Furthermore, leptin decreases glucose, recovers insulin resistance, diminishes hyperlipidemia (Havel, 2002), and stimulates lipolysis through central and peripheral pathways involving AMPK (Ahima, 2006). AMPK switches off pathways of adenosine triphosphate (ATP) consumption and switches on the ATP production pathways, such as fatty acid oxidation and glucose uptake. Moderated AMPK activity in the liver, muscle, adipose tissue, and other peripheral tissues is a predisposing factor for obesity and diabetes as it reduces one's exercise capacity and glucose tolerance (Stark, Ashley, Andrews, 2013).

Lupine (Lupinus albus), a member of the Fabaceae family, is located around the Mediterranean Sea region, especially in North Africa and along the Nile River. Lupine's seed contains protein, lipids, fatty acids, amino acids, carbohydrates, and some minerals such as copper, phosphorus, calcium, and manganese. In addition, the plant contains various amounts of vitamin E, vitamin C, thiamin, riboflavin, and niacin (Lampart-Szczapa et al., 2003a). Lupine seeds contain up to $2 \%$ of quinolizidine alkaloids such, as lupinine and gramine, which are known to be toxic to humans and animals; however, repetitive soaking in water in room temperature (debittering) allows such poisonous alkaloids to be eliminated (ElShazly, Ateya, Wink, 2001). Lupine seeds feature many proven pharmacological and medicinal activities, such as hypoglycemic (Sheweita et al., 2002), hypotensive, and diuretic activities (Medhin et al., 1986). Lupine seed extract also exhibits anti-bacterial properties due to the presence of phenolic compounds (Lampart-Szczapa et al., $2003 \mathrm{~b}$ ). The seed can produce up to $12 \%$ of its total weight as oil, containing components of different saturated and unsaturated fatty acids. The fatty acid composition of the lupine seed oil renders oleic acid as the most abundant fatty acid (40\%-50\%), followed by linoleic acid ( $8 \%-16 \%)$, and $\alpha$-linolenic acid (5\%-10\%) (Sbihi et al., 2013). Although the pharmacological and medicinal activities of lupine seeds are well recognized, there are few reports that discuss the oil. Lupine oil has been used in the development of several cosmetic and topical preparations as anti-wrinkle, sunscreen, and skin protection agents (Sipsas, 2008). However, there is almost no literature discussing the medicinal use of lupine oil. The influence of lupine-enriched foods on obesity and related cardiovascular diseases is well scrutinized; nevertheless, all research attributed this activity to the protein and fibrous parts of the seed (Belski et al., 2011). The effect of lupine oil on such syndromes has never been investigated.

Emulsions, both oil in water $(\mathrm{o} / \mathrm{w})$ or water in oil (w/o), exist in pharmaceutical markets to overcome the formulation difficulties usually associated with oily products, such as bitter taste, moderated solubility, weak absorption, and poor bioavailability (Savjani, Gajjar, Savjani, 2012). For instance, the absorption of crude fish oil was enhanced by $300 \%$ via an emulsification process (Raatz et al., 2009); yet stability-related obstacles in the emulsified products, such as creaming, cracking, and phase separation, could represent a great drawback during their formulation (Kamba, Itodo, Ogah, 2013). Nanoemulsion has recently attracted much attention in pharmaceutical research for its capability to reduce the drawbacks associated with regular emulsion. Nanoemulsion is an emulsion process featuring particle sizes $<1,000 \mathrm{~nm}$, obtained through two successive homogenization processes: primary and secondary. The primary homogenization process aims to mix the immiscible phases with the aid of an emulsifying agent, while the secondary homogenization process decreases the size of the emulsion droplet particles to nanoscale (Sharma et al., 2013).

Obesity and its related ailments are increasing worldwide, and numerous weight-loss drugs are associated with many safety concerns. The aim of this study is to propose lupine oil, both crude and nanoemulsion formulations, as a natural medication for obesity and its related complications by investigating the effect of the oil on different physiological parameters and hormone levels.

\section{MATERIAL AND METHODS}

\section{Chemicals and reagents}

Sunflower oil was purchased from Al-Ahlam Company for seed oil production (Jeddah, Saudi Arabia). Tween 20 was purchased from Sigma-Aldrich Co. (St Louis, MO, USA). Leptin rat enzyme-linked immunosorbent assay (ELISA) kit (ab100773) and adiponectin rat ELISA kit (ab108784) were obtained from Abcam plc (Cambridge, UK). A rat insulin ELISA kit (80-INSRT-E01) was purchased from ALPCO Diagnostics (Salem, NH, USA). AMPK $\alpha 1 / 2$ 
(SC-25792), ACRP30, leptin (SC-842), $\quad \beta$-actin (SC-130656), goat anti-rabbit immunoglobulin (Ig) G-horseradish peroxidase (HRP) (SC-2030) antibodies, luminol reagent (SC-2048), and polyvinylidene fluoride (PVDF) membrane (SC-3723) were purchased from Santa Cruz Biotechnology, Inc. (Dallas, TX, USA). Alanine aminotransferase (ALT), blood glucose, total cholesterol, and high-density lipoprotein (HDL)cholesterol kits were purchased from United Diagnostic Industry (Dammam, KSA). The remaining chemicals used were of analytical grade and obtained from a local distribution agent in KSA.

\section{Plant materials}

The seeds of sweet white lupine (Lupinus albus, family Fabaceae) were collected from a local farm near Cairo, Egypt in May 2014 when the plant was in its flowering and fruiting stage. The whole plant was identified by Dr. AH Mohamed, plant taxonomist, Egyptian Agricultural Museum, Cairo, Egypt. Voucher specimens were deposited in the herbarium of the Department of Pharmacognosy, Faculty of Pharmacy, Zagazig University, Egypt, under number F352.

\section{Isolation of lupine oil}

Lupine seeds were debittered to remove toxic alkaloids according to (Erbas, 2010), as follows: the lupine seeds were soaked in water for 12 hours, and they were then boiled for 1 hour (a ratio of 1:3 of seeds: water $[\mathrm{w} / \mathrm{v}])$ to soften the seeds. The boiled seeds were debittered by soaking them again in tap water for 48 hours at room temperature $\left(25^{\circ} \mathrm{C}\right)$; the debittering water was changed every 2 hours. The soaked seeds were allowed to dry; they were then powdered using a plant grinder (IKA-M20). A total of $250 \mathrm{~g}$ of powder were extracted using $1 \mathrm{~L}$ of $n$-hexane in a Soxhlet apparatus for 6 hours at $70{ }^{\circ} \mathrm{C}$. The organic solvent was evaporated under reduced pressure to obtain the crude oil $(9.4 \%$ yield). The oil samples were kept in a refrigerator at $4{ }^{\circ} \mathrm{C}$ until further use. Crude lupine oil was diluted using sunflower oil to reach the required concentration for administration to the animals.

\section{Preparation of the nanoemulsion}

Nanoemulsions were formulated with different ratios, as detailed in the table below. Crude lupine oil, diluted with sunflower oil, was vortexed with Tween 20 for 10 minutes at 2,000 rpm; then, the aqueous phase was added slowly with continuous mixing for an extra 10 minutes until a milky white emulsion was obtained. The second homogenization was performed with a homogenizer (IKA-T25; Germany) at 10,000 rpm for 5 minutes to produce four nanoemulsion formulas with different ratios, as outlined in table I. The produced nanoemulsions were stored in tightly sealed glass containers until further analysis.

TABLE I - Composition of the prepared lupine nanoemulsion

\begin{tabular}{lcccc}
\hline Formula & $\begin{array}{c}\text { Lupine } \\
\text { (g) }\end{array}$ & $\begin{array}{c}\text { Sunflower } \\
\text { oil (g) }\end{array}$ & $\begin{array}{c}\text { Distilled } \\
\text { water (g) }\end{array}$ & $\begin{array}{c}\text { Tween 20 } \\
\text { (g) }\end{array}$ \\
\hline F1 & 0.25 & 1.05 & 2.70 & 1 \\
F2 & 0.25 & 1.25 & 2.50 & 1 \\
F3 & 0.25 & 1.45 & 2.30 & 1 \\
F4 & 0.25 & 1.65 & 2.10 & 1 \\
\hline
\end{tabular}

\section{Characterizations of nanoemulsions}

\section{Particle size determination}

A total of $10 \mu \mathrm{L}$ of the prepared nanoemulsions were diluted with $3 \mathrm{~mL}$ of distilled water; their particles sizes were determined through a Malvern zetasizer (Nanoseries, zs; Malvern Instruments, Malvern, UK).

\section{Viscosity determination}

The viscosity of the prepared nanoemulsions was determined through a Brookfield viscometer (DV-II Pro; USA). The dynamic viscosity measurements were determined using spindle No. 64 and $60 \mathrm{rpm}$ at room temperature.

\section{Investigation of phase separation}

The prepared nanoemulsions were kept for 3 months at room temperature; the phase separation was investigated and photographically recorded. 


\section{Animals}

Thirty male Sprague-Dawley rats weighing $(170 \pm 25 \mathrm{~g})$ were obtained from the College of Veterinary Medicine and Animal Resources, King Faisal University, Hofuf, KSA. The rats were kept in standard laboratory conditions $\left(23{ }^{\circ} \mathrm{C} \pm 1{ }^{\circ} \mathrm{C}\right)$ and maintained on a standard commercial rodent diet using a 12-hour light/dark cycle throughout the accommodation period (1 week).

\section{In vivo experimental design}

All animal experimental procedures and protocols were approved by the Animal Research Ethics Committee at King Faisal University, and they were performed in accordance with the Guidelines for the Ethical Conduct for Use of Animals in Research, King Faisal University. Rats were divided into two groups after acclimating to the facility: one group $(n=5)$ was continued on a standard commercial rodent diet and served as the non-obese control (NO). The other group $(n=25)$ was fed a high-fat diet (HFD; a rodent diet with $60 \%$ of its energy provided from fat; Research Diet, New Brunswick, NJ, USA) for 7 weeks to prepare the obese animal model. After 7 weeks, the obese rat group was divided into five groups: untreated obese rats (obese); obese rats that received $100 \mathrm{mg} / \mathrm{kg}$ of the crude lupine oil diluted in sunflower oil for 10 days (L10) or 20 days (L-20); and obese rats that received 100 $\mathrm{mg} / \mathrm{kg}$ of the lupine oil in nanoemulsion formulation for 10 days (NL-10) or 20 days (NL-20). Untreated control obese rats (obese group) have received an equal volume of sunflower oil as a vehicle of lupine crude oil doses or nanoemulsion formulations. All obese rats were fed the HFD during the treatment period, and all formulations were administered by oral gavage. By the end of each designated time, the rats were sacrificed and their blood was collected, as were their liver tissues and epididymal fat. The serum was separated by centrifugation of the blood at 3,000 rpm for 10 minutes, and samples were stored at temperatures of $-20{ }^{\circ} \mathrm{C}$ until analysis.

\section{Determination of physiological and biochemical parameters}

Blood glucose, serum insulin, ALT, total cholesterol, and HDL levels were measured by their corresponding kits, as mentioned under the chemicals and reagents section, and according to manufacturer protocols. Food intake by all rats was determined according to Jeong et al. (2016), where the food intake of the obese group was considered to be $100 \%$, and the food intake of the other groups was calculated every 3 days. The body weight (g) and epididymal fat weight (g) of all groups were also determined as biochemical parameters.

\section{Hormonal determination}

Leptin and adiponectin were determined in serum using the kits described under the chemical and reagent section and according to manufacturer protocols. Leptin, adiponectin, and AMPK expression were determined in the epididymal fat by Western blot analysis, as will be described. Similarly, AMPK was also determined in the liver homogenate by Western blot analysis. The blot analysis was performed as described by (Takasawa et al., 2008). Briefly, liver or epididymal fat samples were homogenized in radioimmunoprecipitation assay (RIPA) buffer with a protease inhibitor; the total protein extracted from those homogenates was quantified using a NanoDrop Lite spectrophotometer (Thermo Fisher Scientific, Waltham, MA, USA). Then, $50 \mu \mathrm{g}$ of the total extracted protein was separated by sodium dodecyl sulfate (SDS)-polyacrylamide gel electrophoresis (PAGE) and blotted onto PVDF membranes. The membranes were blocked by incubation in Tris-buffered saline (TBS) containing 3\% bovine serum albumin and $0.1 \%$ Tween 20 for 1 hour at room temperature. After washing with TBS containing $0.1 \%$ Tween 20 , the membranes were incubated with the primary antibodies (1:300 dilution) for 2 hours at room temperature, and they were then incubated with the secondary antibody (goat anti-rabbit HRP-conjugated at a 1:5,000 dilution). The chemiluminescence produced from the luminol reagent was detected with the LI-COR C-DiGit chemiluminescence scanner, and the intensity of the bands was analyzed using the scanner software. In the epididymal fat experiment, loading uniformity was assessed by Ponceau S staining of the membranes after blotting given the low expression of $\beta$-actin in the adipose tissue.

\section{Statistical analysis}

Data were expressed as the mean \pm standard deviation (SD) of at least three independent experiments 
$(n=5)$. Statistically significant differences between the groups were determined by means of one-way analysis of variance (ANOVA) followed by Tukey's multiple comparison test using IBM SPSS software (IBM SPSS Statistics for Windows (2010), Version 19.0. Armonk, NY: IBM Corp). In all cases, probability values of $\mathrm{P}<0.05$ were taken as statistically significant.

\section{RESULTS}

Crude lupine oil was extracted from the plant seeds after a debittering process in which toxic alkaloids were eliminated. The crude oil was diluted to the required dose, for either animal administration or the nanoemulsion formulation, by sunflower oil.

\section{Pharmaceutical formulation of lupine oil}

The sunflower oil-diluted crude lupine oil (L) underwent emulsification with further particle size reduction to produce the nanoemulsion. Four nanoemulsion formulas (F1-F4) were prepared with different ratios, as mentioned previously, and in all cases, milky white nanoemulsions were obtained with no signs of phase separation.

\section{Effect of nanoemulsion composition on particle size}

The particle sizes of the four prepared nanoemulsions showed good correlations with the percentage of the oil phase, as shown in Table II. Increasing the oily phase from F1 to F4 resulted in increasing particle sizes from $261 \mathrm{~nm}$ to $373 \mathrm{~nm}$, respectively. The particle size distribution analysis of the nanoemulsion formula $\mathrm{F} 4$ displayed a polydispersity index (PDI) equal to 0.291, indicating good homogeneity (Figure 1A).

TABLE II - Particle size distribution analysis, viscosity, and phase separation of the prepared nanoemulsion formulations

\begin{tabular}{lccc}
\hline Formula & $\begin{array}{c}\text { Particle } \\
\text { size (nm) }\end{array}$ & Viscosity (cp) & $\begin{array}{c}\text { Phase } \\
\text { separation }\end{array}$ \\
\hline F1 & 261 & 240 & $+\mathrm{ve}$ \\
F2 & 305 & 469 & $+\mathrm{ve}$ \\
F3 & 347 & 535 & $+\mathrm{ve}$ \\
F4 & 373 & 659 & $-\mathrm{ve}$ \\
\hline
\end{tabular}
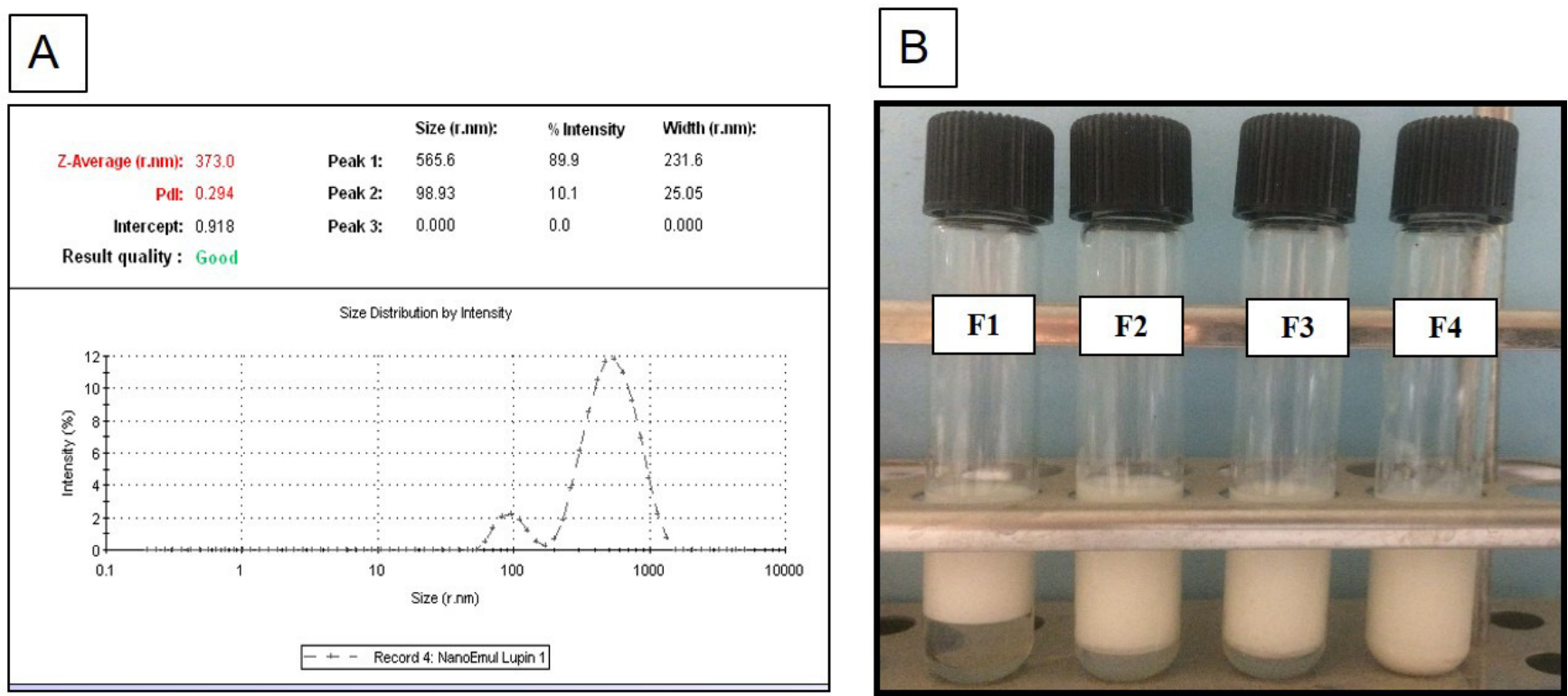

FIGURE 1- Characterization of lupine oil nanoemulsion: (a) Droplet size distribution of the formula F4. (b) Photograph of different nanoemulsion preparations after 3 months storage at room temperature. 


\section{Effect of nanoemulsion composition on viscosity}

Table II indicated that the viscosity of the prepared nanoemulsion was $240 \mathrm{cp}, 469 \mathrm{cp}, 535 \mathrm{cp}$, and $659 \mathrm{cp}$ for formulations F1, F2, F3, and F4 respectively.

\section{Effect of storage on nanoemulsion phase separation}

The prepared nanoemulsion formula F4 showed good stability; however, other formulations F1, F2, and F3 suffered from phase separation after being stored on a shelf for 3 months at room temperature (Figure 1B). According to the previously mentioned data, the F4 nanoemulsion (NL) and the sunflower oil-diluted crude lupine oil (L) formulations were administered to the animals to investigate their physiological, biomedical, and hormone profiles.

\section{Effect of lupine oil formulations on some physiological and biochemical parameters}

The rats were divided into one of six groups; five of them were rendered obese by the administration of a HFD, and the remaining group served as the non-obese control. Both oil formulas (crude and nanoemulsion) were dispensed orally to the rats, and all results were taken in comparison with the untreated obese group (obese) unless otherwise specified.

\section{Effect of lupine oil on liver enzymes}

To test the effect of lupine oil on liver integrity, the ALT level, which is one of the most important liver integrity enzymes, was ascertained. Administering the lupine oil did not significantly alter the ALT levels at any time point or for any formula (Table III).

\section{Effect of lupine oil on blood glucose and insulin levels}

Table III showed a significant $(\mathrm{P}<0.05)$ effect of lupine oil nanoemulsion on fasting blood glucose and insulin levels in the (NL-20) group, although the level of both biomarkers increased, but not significantly so, by the induction of an obese state.

\section{Effect of lupine oil on the lipid profile and epididymal fat}

Feeding rats with a HFD for 7 weeks significantly $(\mathrm{P}<0.001)$ elevated their total cholesterol levels and decreased their HDL levels when compared with the non-obese control group (Table III). Administration of the crude lupine oil for 10 days (L-10) had no

TABLE III - Physiological and biochemical parameters for the different groups and time points: none-obese (NO) obese untreated (obese), Lupine crude oil treated after 10 days (L-10) and 20 days (L-20), and lupine oil nanoemulsion formulation after 10 days $(\mathrm{NL}-10)$ and 20 days (NL-20). Values are mean $\pm \mathrm{SD}(\mathrm{n}=5)$

\begin{tabular}{lcccccc}
\hline Parameters & NO & Obese & L-10 & NL-10 & L-20 & NL-20 \\
\hline ALT (U/L) & $45.25 \pm 4.11$ & $47.25 \pm 3.3$ & $46 \pm 2.71$ & $50 \pm 3.91$ & $47.5 \pm 4.8$ & $49.25 \pm 2.2$ \\
Fasting blood glucose (mg/dl) & $86.75 \pm 2.75$ & $89.5 \pm 6.45$ & $83.25 \pm 3.1$ & $82.25 \pm 4.34$ & $81.5 \pm 4.65$ & $80.5 \pm 3.1$ \\
Insulin (ng/ml) & $1.39 \pm 0.09$ & $1.5 \pm 0.07$ & $1.34 \pm 0.11$ & $1.27 \pm 0.08$ & $1.29 \pm 0.1$ & $1.19 \pm 0.02^{*}$ \\
Total cholesterol (mmol/L) & $2.77 \pm 0.12^{* * *}$ & $3.68 \pm 0.09$ & $3.52 \pm 0.15$ & $3.29 \pm 0.09^{*}$ & $3.20 \pm 0.11^{* *}$ & $3.06 \pm 0.11^{* * *}$ \\
HDL-cholesterol (mmol/L) & $1.52 \pm 0.05^{* * *}$ & $1.19 \pm 0.07$ & $1.22 \pm 0.03$ & $1.27 \pm 0.07$ & $1.37 \pm 0.04^{*}$ & $1.42 \pm 0.09^{* *}$ \\
Epididymal fat (gm) & $1.9 \pm 0.15^{* * *}$ & $5.66 \pm 0.86$ & $5.32 \pm 0.66$ & $4.58 \pm 0.63^{*}$ & $3.92 \pm 0.65^{* *}$ & $3.56 \pm 0.51^{* * *}$ \\
Body weight (gm) & $219 \pm 12.67 * * *$ & $284.5 \pm 17.15$ & $274 \pm 15.07$ & $270 \pm 9.41$ & $257 \pm 10.8$ & $249.5 \pm 10.53^{* *}$ \\
Food intake (gm) & $101 \pm 2.64$ & $100 \pm 1.00$ & $93.3 \pm 3.51$ & $86 \pm 5.29^{*}$ & $81.33 \pm 3.2^{* *}$ & $77.66 \pm 6.8^{* * *}$ \\
\hline
\end{tabular}

Notes: Abbreviations $* \mathrm{P}<0.05, * * \mathrm{P}<0.01$ and $* * * \mathrm{P}<0.001$ compared with (Obese) values. 
significant effect on the level of total cholesterol; however, formulating the oil into a nanoemulsion $(\mathrm{NL}-10)$ significantly $(\mathrm{P}<0.05)$ reduced the level of total cholesterol in obese rats (Table III). Furthermore, administering both oil formulations (L-20 and NL20) for 20 days induced a significant $(\mathrm{P}<0.01$ and $\mathrm{P}<0.001$, respectively) reduction in total cholesterol levels in obese rats (Table III). Additionally, lupine oil significantly $(\mathrm{P}<0.05$ and $\mathrm{P}<0.01)$ increased the levels of HDL-cholesterol after 20 days (L-20 and NL-20), respectively; however, no significant alterations were observed after 10 days of the oil administration. Table III indicates that there was a significant $(\mathrm{P}<0.001)$ increase in epididymal fat weight following administration of the HFD in comparison to the non-obese control group. This fat accumulation is significantly $(\mathrm{P}<0.05)$ reduced following the administration of the lupine oil nanoemulsion for 10 days, or after the administration of both oil formulations (L-20, $\mathrm{P}<0.01$; and NL-20, $\mathrm{P}<$ 0.001 , respectively) for 20 days (Table III).

\section{Effect of lupine oil on body weight and food intake in the HFD-induced obesity model}

The HFD induced a significant $(\mathrm{P}<0.001)$ increase in the rats' body weight when compared to the non-obese rats, although the amount of food intake in both groups was similar with no significant difference (Table III). After 10 days of lupine oil administration, no significant difference was detected in the rats' body weight for either of the two oil formulations; however, the lupine oil nanoemulsion (NL-10) reduced the amount of food intake significantly $(\mathrm{P}<0.05)$ (Table III). After 20 days of the oil administration, the crude oil formulation (L20) still did not yield a significant change in the rats' body weight; however, when the oil was formulated into the nanoemulsion (NL-20), a significant $(\mathrm{P}<0.01)$ reduction in the rats' body weight was found. Both oil formulations (L-20 and NL-20) significantly reduced the amount of food intake by the obese rats $(\mathrm{P}<0.01$ and $\mathrm{P}<0.001$, respectively) (Table III).

\section{Effect of lupine oil on rats' hormone profile}

Figure 2A shows a significant $(\mathrm{P}<0.01)$ increase in the leptin expression levels after 10 and 20 days of crude oil administration (L-10 and L-20) in the epididymal fat by $1.39 \pm 0.08$-fold and $1.37 \pm 0.10$-fold, respectively. This increase in leptin expression was amplified when the nanoemulsion formulations (NL10 and NL-20) were used to reach expression levels of $1.73 \pm 0.14$-fold and $1.92 \pm 0.12$-fold, respectively. This noteworthy increase in leptin levels was not limited to the epididymal fat; rather, it extended to the serum level of the hormone as well, where a significant difference $(\mathrm{P}<0.05)$ can be observed in leptin expression level after 10 days of oil administration in the nanoemulsion formulation (Figure 2B). However, after 20 days of oil administration, both formulations were associated with a significant elevation of leptin expression with P-values $<0.05$ and $<0.001$, respectively (Figure $2 \mathrm{~B}$ ). In all cases, the nanoemulsion formulation showed a significant $(\mathrm{P}<0.001)$ increase in leptin expression levels when compared to the corresponding crude oil formulation (Figure 2).

A significant $(\mathrm{P}<0.01$ and $\mathrm{P}<0.001)$ decrease in adiponectin levels in both epididymal fat and serum can be observed following the induction of obesity (Figure 3). The low adiponectin levels not only reverted back to the normal levels, but they were also elevated and exceeded the normal reading as well; this was the case for both the epididymal fat and serum following the administration of lupine oil. Western blot analysis revealed the over-expression of adiponectin in the epididymal fat following treatment with lupine oil by $1.7 \pm 0.15$-fold, $3.8 \pm 0.133$-fold, $5.1 \pm 0.42$-fold, and $9.6 \pm 1.52$-fold in the L-10, NL-10, L-20, and NL-20 groups, respectively (Figure 3A). Only the lupine oil nanoemulsion was able to cause a significant $(\mathrm{P}<0.05)$ increase in the adiponectin serum levels following 10 days of administration; however, both formulations were able to induce the same effect after 20 days (Figure $3 B)$. Generally, the effect of the nanoemulsion was more pronounced and caused a nearly 10 -fold increase in adiponectin levels in the epididymal fat after 20 days of formulation administration (Figure 3).

Western blot analysis was performed on both liver and epididymal fat homogenates to investigate the effect of lupine oil on AMPK expression. The analysis of epididymal fat AMPK levels revealed a sharp and significant $(\mathrm{P}<0.001)$ increase in the AMPK expression in all groups except L-10, which was nearly 15 -fold greater following the administration of the lupine oil nanoemulsion formulation for 20 days (Figure 4A). Similarly, a significant over-expression of AMPK was detected in the liver homogenate following oil administration with $\mathrm{P}<0.001$ for the NL-10, L-20, and NL-20 groups and $\mathrm{P}<0.01$ for the L-10 group (Figure 


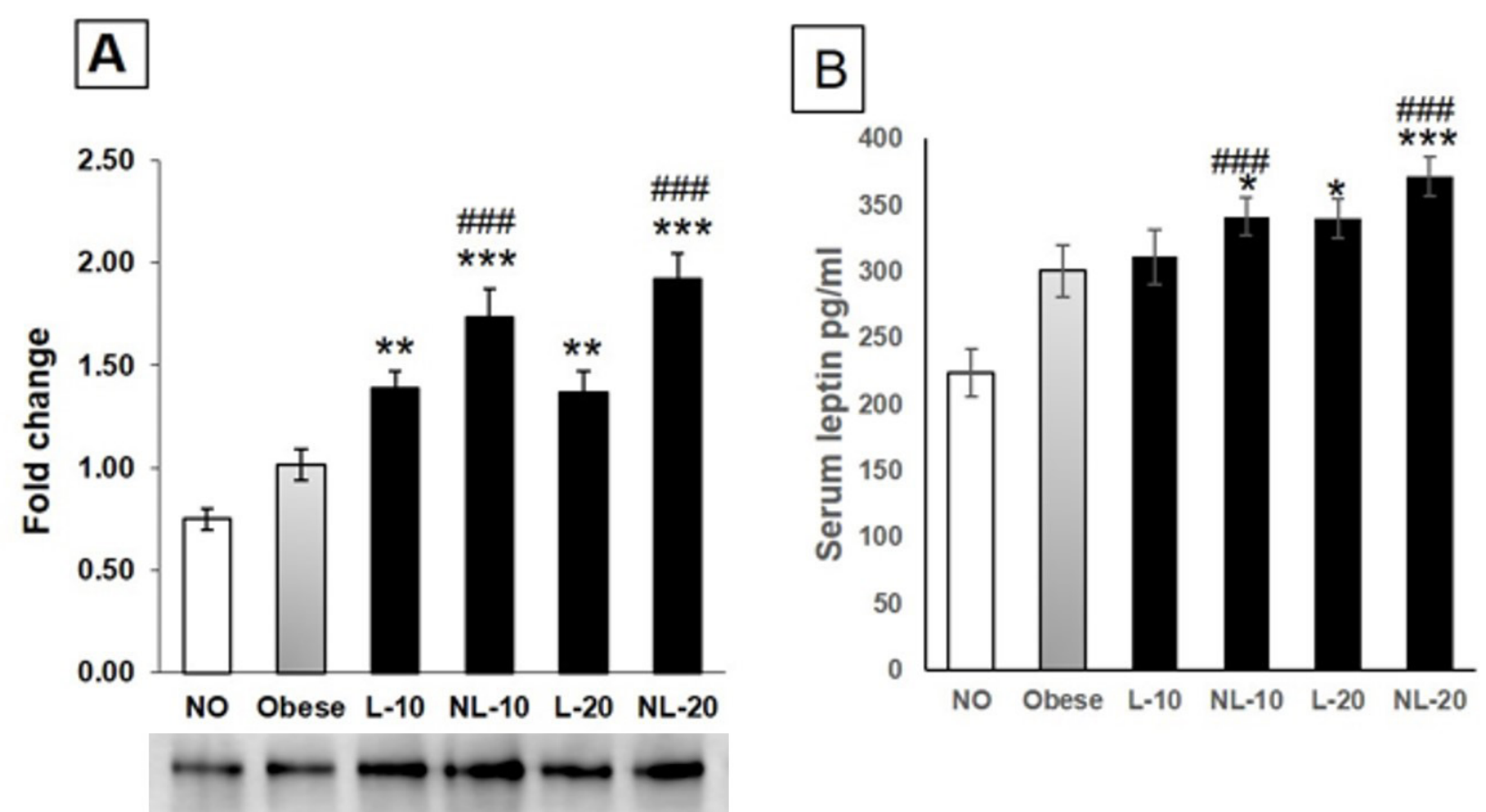

FIGURE 2- Effect of lupine oil on leptin expression: (a) Leptin expression determined by Western blot analysis in the epididymal fat. (b) Serum level of leptin determined by ELISA. NO: non-obese group, Obese: obese untreated group, L-10 and L-20: crude lupine oil treated groups after 10 and 20 days, NL-10 and NL-20: Lupine oil nanoemulsion treated groups after 10 and 20 days. Data are represented as means $\pm \mathrm{SD},(n=5) . * \mathrm{P}<0.05, * * \mathrm{P}<0.01$ and $* * * \mathrm{P}<0.001$ when compared with obese untreated values. $\# \mathrm{P}<0.05$, \#\# $\mathrm{P}<0.01$ and \#\#\# $\mathrm{P}<0.001$ when crude oil preparation group is compared to its corresponding nanoemulsion formulation group (L-10 versus NL-10 and L-20 versus NL-20).

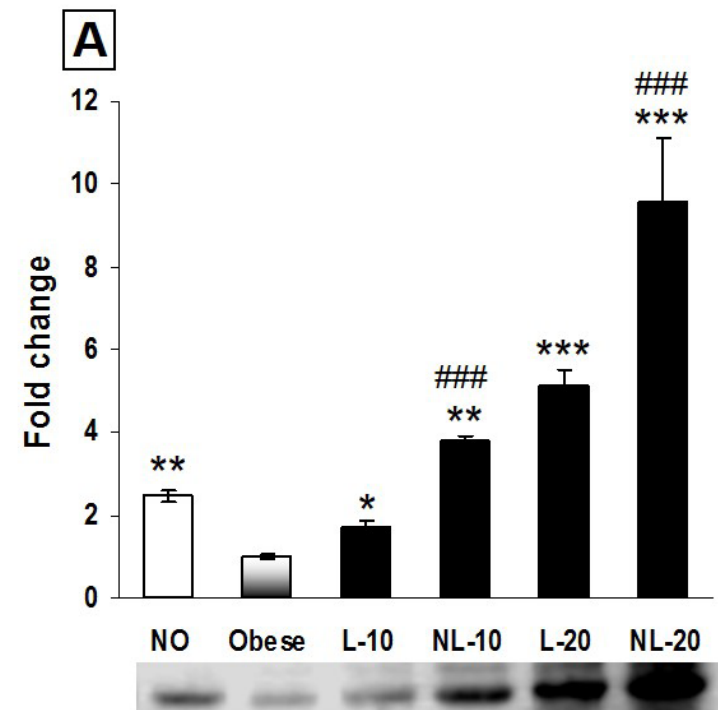

adiponectin

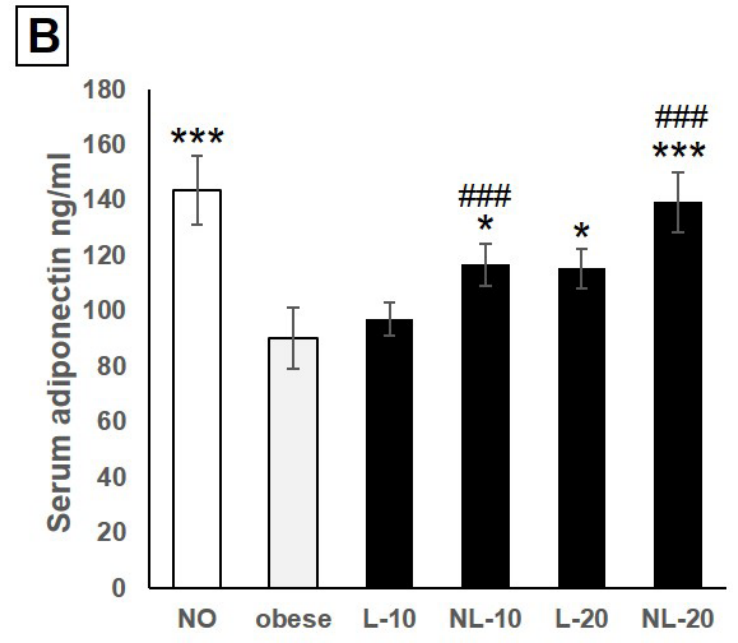

FIGURE 3- Effect of lupine oil on adiponectin expression: (a) adiponectin expression determined by Western blot analysis in the epididymal fat. (b) Serum level of adiponectin determined by ELISA. NO: non-obese group, Obese: obese untreated group, L-10 and L-20: crude lupine oil treated groups after 10 and 20 days, NL-10 and NL-20: Lupine oil nanoemulsion treated groups after 10 and 20 days. Data are represented as means $\pm \mathrm{SD},(n=5)$. $* \mathrm{P}<0.05, * * \mathrm{P}<0.01$ and $* * * \mathrm{P}<0.001$ when compared with obese untreated values. \# $\mathrm{P}<0.05$, \#\# $\mathrm{P}<0.01$ and \#\#\# $\mathrm{P}<0.001$ when crude oil preparation group is compared to its corresponding nanoemulsion formulation group (L-10 versus NL-10 and L-20 versus NL-20). 
4B). Comparable to what was observed for leptin and adiponectin, the lupine oil nanoemulsion formulation (NL-10 and NL-20) showed more intense activation for AMPK following administration for either 10 days or 20 days (Figure 4).

The consumption of excess nutrients with high caloric values is responsible for the widespread obesity in developing countries and around the world. Many common metabolic complications are primarily ascribed to obesity, including dyslipidemia, insulin resistance, diabetes, and hypertension (Deng, Scherer, 2010). Anti-obesity drugs are important adjunctive therapy, in combination with lifestyle changes, to improve the obesity status of obese individuals. Unfortunately, many of the reported antiobesity drugs are associated with many limitation due to their high attrition rates, their burden on the liver, and the lack of long-term morbidity and mortality data (Padwal, Majumdar, 2007). In light of the aforementioned data on the obesity pandemic, the development of safe and effective treatment alternatives should be a priority. This study investigates lupine oil as an anti-obesity drug, which is in accordance with the line of alternative developments of natural and safe weight-loss agents.

In the present study, the effect of different formulations of lupine oil on HFD-induced obesity was investigated. Treatment with lupine oil significantly decreased rats' food intake, epididymal fat, and body weight, and improved their lipid profile levels; it also modulated the rats' blood glucose levels and insulin resistance. This anti-obesity effect of lupine oil was associated with the increased expression of both adiponectin and leptin, and this effect may be mediated through AMPK activation. Additionally, the oil and its formulations did not have any effects on the liver's integrity, as was proven by the non-altered ALT levels.

Obesity is characterized by dyslipidemia and the accumulation of fat in the abdominal region. Table III illustrates that there was a reduction in the rats' body weight and epididymal fat weight. This reduction is associated with reduced food intake, and it may be influenced by the rats' decreased appetite for food intake. This could be attributed to increased levels of lipolysis by the administration of lupine oil. Oleic acid is the primary fatty acid component in lupine seed oil (40\%-50\%), followed by linoleic acid $(8 \%-16 \%)$ and $\alpha$-linolenic acid (5\%-10\%) (Sbihi et al., 2013). Oleic acid was proven to have an anorectic effect, which is independent of leptin (Obici et al., 2002). Moreover, $\alpha$-linolenic is evidenced to have an inhibitory effect on lipogenesis and thus exhibits hypolipidemic activity $(\mathrm{Su}$
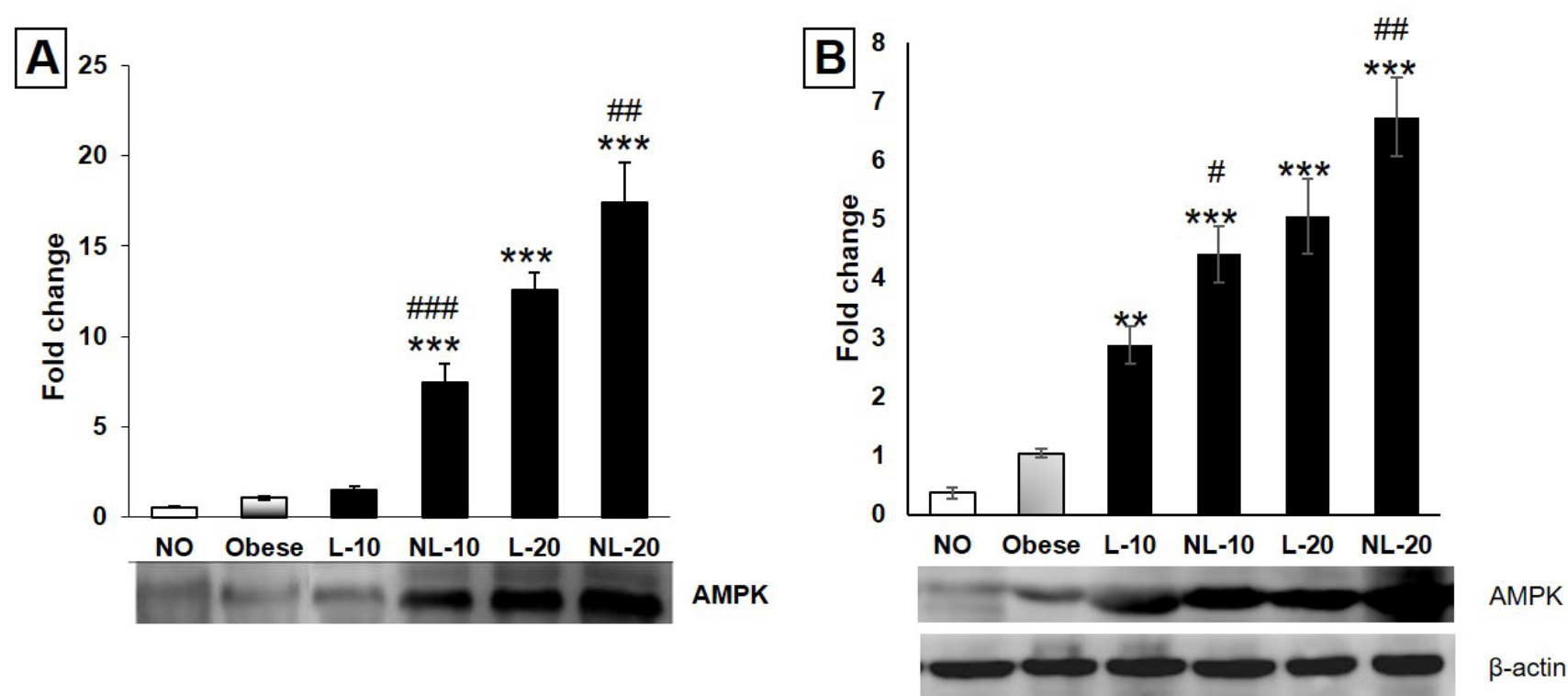

FIGURE 4- Effect of lupine oil on AMPK expression: (a) Expression of AMPK in epididymal fat determined by Western blot analysis. (b) Expression of AMPK in liver homogenate determined by Western blot analysis. NO: non-obese group, Obese: obese untreated group, L-10 and L-20: crude lupine oil treated groups after 10 and 20 days, NL-10 and NL-20: Lupine oil nanoemulsion treated groups after 10 and 20 days. Data are represented as means $\pm \mathrm{SD},(n=5)$. $* \mathrm{P}<0.05, * * \mathrm{P}<0.01$ and $* * * \mathrm{P}$ $<0.001$ when compared with obese untreated values. \# $\mathrm{P}<0.05$, \#\# $\mathrm{P}<0.01$ and \#\#\# $\mathrm{P}<0.001$ when crude oil preparation group is compared to its corresponding nanoemulsion formulation group (N-10 versus NL-10 and L-20 versus NL-20). DISCUSSION 
et al., 2016). Lupine seeds are known to reduce food intake by $30 \%$, as they decrease appetite; however, this effect has been attributed to the protein and carbohydrate parts of the seed (Belski et al., 2011). Nevertheless, this study suggests that lupine seed oil plays this role. Lupine oil significantly reduced the total cholesterol levels and increased the HDL levels in a time- and formulationdependent manner. This may be attributed to gene expression regulation, particularly among those genes involved in lipid metabolism; however, this speculation requires further investigation.

Leptin and adiponectin are adipocyte hormones that control energy homeostasis (Havel, 2002). Leptin, a pleiotropic hormone, plays a role in appetite suppression and it increases energy expenditure (Mantzoros et al., 2011). It acts as a signal of the body's nutritional status to the reproductive, immune, cardiovascular, skeletal, and thyroid systems. Moreover, it plays a regulatory role in blood sugar levels through its effects on the liver, pancreas, and blood pressure, both directly and via the sympathetic nervous system (Nasrallah, Ziyadeh, 2013). The present study showed that lupine oil not only maintained the elevations in serum leptin necessary for decreasing rats' appetite for food intake, but the oil also increased the expression of leptin in the epididymal fat. The increased level of leptin has a direct effect on the hypothalamus, which reduces one's appetite and subsequently decreases food intake; this may explain the reduction in body weight observed in the present study.

Leptin and adiponectin tend to normalize insulin action through AMPK-induced fat oxidation, leading to reduced triglyceride content intramyocellulary and in the liver (Meier, Gressner, 2004; Galic, Oakhill, Steinberg, 2010). Adiponectin has been linked to glucose, lipid, and cardiovascular regulation (Ahima, 2006; Akingbemi, 2013). A deficiency of adiponectin and/or decreased adiponectin receptormediated activity contribute to insulin resistance, hyperinsulinemia (Meier, Gressner, 2004), liver disease, and coronary heart disease (Akingbemi, 2013). Adiponectin has an insulin-sensitizing effect, and it also exerts its effects on metabolism of the lipid profile (Lara-Castro et al., 2007). Adiponectin is an attractive therapeutic target for the prevention of cardiovascular diseases and diabetes, as it can be significantly increased by diet, exercise, and weight loss (Silva, de Almeida, Feoli, 2011). The elevation of adiponectin levels led to increased insulin sensitivity, enhanced fatty acid oxidation, and increased energy expenditure, and it also reduced the production of glucose by the liver (Galic, Oakhill, Steinberg, 2010). The results clearly show the reduced expression of adiponectin in the untreated obese group; moreover, the lupine oil can induce over-expression of the hormone in the epididymal fat, as well as in the serum, at all time points and, in part, with all formulations. This elevation in adiponectin level may explain the reduced blood glucose and insulin levels, as well as the ameliorated lipid profile. Diets containing $\alpha$-linolenic or oleic fatty acids, which are the main components of lupine oil, improved the state of insulin resistance in obese mice (Oliveira et al., 2015); the present study may explain the mechanism of such improvement through the over-expression of adiponectin in both epididymal fat and serum.

Activated AMPK, through high levels of adiponectin, stimulates fatty acid oxidation, mitochondrial biogenesis, and glycolysis, and it also inhibits glycogen, fatty acid, and protein synthesis and gluconeogenesis; it also has a direct appetite-regulating effect in the hypothalamus (Lim, Kola, Korbonits, 2010). The results of this study indicated that lupine oil increased the expression of both leptin and adiponectin, and this was concomitant with the over-expression of AMPK in both the epididymal fat (at some points, to about 20 -fold) and liver, implying that lupine oil activity is associated with the AMPK pathway.

Numerous lipophilic drugs demonstrate low oral bioavailability due to their poor aqueous solubility (Gershanik, Benita, 2000). Lupine oil has a lipophilic character and it may be hindered by its low bioavailability due to its interrupted absorption. Nanoemulsions are submicron-sized dosage forms that area associated with various advantages, including their enhanced drug solubility and bioavailability, increased drug loading, and controlled drug release (Choi et al., 2010; Sharma et al., 2013). Accordingly, lupine oil was formulated into a nanoemulsion to overcome any absorption limitations and to ensure enhanced oil bioavailability. The results stated that nanoemulsion formulations with particle sizes $<400 \mathrm{~nm}$ were obtained and exhibited good viscosities, which will not affect the stability or oral administration aptitude of the prepared formulation. As the oil phase concentration increases, the viscosity of the prepared nanoemulsion increases (Table II), and these results are in accordance with those reported previously (Dłu ewska, Stabiecka, Maszewska, 2006). 
Nanoemulsion formulation F4 showed good stability over 3 months at room temperature, so it was selected for further in vivo investigations in comparison to crude lupine oil. The administration of the lupine oil nanoemulsion formulation intensified the physiological, biochemical, and hormonal activity of the oil in all cases and at all time points. The nanoemulsion formulation of the oil showed a significant difference in the efficacy of the lupine oil, especially in terms of adiponectin and AMPK levels. In addition to the fact that the nanoemulsion formulation increased the lupine oil's bioavailability, this preparation can also be used as a lipid-based formula that can reduce the limitations associated with the slow and incomplete dissolution of those drugs that are poorly soluble in water; moreover, this preparation can also facilitate the creation of solubilized phases from which absorption may occur (Humberstone, Charman, 1997). This formula can especially be implemented for those drugs that can be used to counteract obesity, diabetes, and hyperlipidemia, so as to take advantage of the synergistic activity between the drug and the formulated lupine oil.

In the presented study, lupine oil showed an antiobesity effect when administered to the HFD-induced obese rats. The oil was able to ameliorate the alterations in the lipid profile caused by the HFD, and it improved both insulin and blood sugar levels as an indication of reduced insulin resistance. Notably, lupine oil reduced both the epididymal fat weight and food intake, which are considered challenges for obese patients. The use of lupine oil was found to be safe, as it did not place any burden on the liver; this is in contrast to many other anti-obesity drugs, which are all associated with hepatic problems. Lupine oil enhanced leptin and adiponectin levels in both the serum and epididymal fat, and it also activated AMPK in both the epididymal fat and liver. The formulation of lupine oil into the nanoemulsion enhanced its activity across all the aforementioned biomarkers, particularly the hormones, indicating that there was enhanced absorption and bioavailability of the formulated oil.

The authors of this study recommend the use of a lupine oil nanoemulsion as a potential anti-obesity agent against diet-induced obesity, which can enhance energy expenditure and reduce appetite, epididymal fat, and body weight without affecting the liver. This recommendation requires further investigations using clinical models to confirm the medicinal properties of this preparation on humans.

\section{ACKNOWLEDGMENTS}

The author thanks the Deanship of scientific research, University of King Faisal for the financial support under the grant number $(2 / 238 / 11)$. The authors acknowledge all the effort exerted by $\mathrm{Mr}$ Tamim Alyahian in preparations, assistance and support in the experimental part of this study. English-language editing of this manuscript was provided by Journal Prep

\section{DECLARATION OF INTEREST}

The authors declare no conflicts of interest in this work.

\section{STATEMENT OF AUTHOR CONTRIBUTIONS TO MANUSCRIPT}

This study was conceptualized and designed by OMM, IAA, TMS and MEM. Lupine and its oil was obtained and extracted by MEM. Nanoemulsion was prepared and characterized by TMS. Animal model was prepared by IAA and OMM. IAA determined physiological and biochemical parameters including Blood glucose, serum insulin, ALT, total cholesterol, and HDL. Hormonal determinations, including Leptin, adiponectin, and AMPK expression was done by OMM. All authors interpreted the data, discussed the results and contributed to preparing the manuscript.

\section{SUBMISSION DECLARATION AND VERIFICATION}

This manuscript has not been published previously and it is not under consideration for publication elsewhere. This manuscript is approved by all authors and by the University of King Faisal where the work was carried out.

\section{REFERENCES}

Ahima RS. Adipose tissue as an endocrine organ. Obesity (Silver Spring). 2006;14(Suppl 5):242s-249s.

Akingbemi BT. Adiponectin receptors in energy homeostasis and obesity pathogenesis. Prog Mol Biol Transl Sci. 2013;114:317-342.

Belski R, Mori TA, Puddey IB, Sipsas S, Woodman RJ, Ackland TR, et al. Effects of lupin-enriched foods on body 
composition and cardiovascular disease risk factors: a 12-month randomized controlled weight loss trial. Int J Obes. 2011;35(6):810-819.

Chang HM, Park HS, Park CY, Song YS, Jang YJ. Association between serum vaspin concentrations and visceral adipose tissue in Korean subjects. Metabolism. 2010;59(9):1276-1281.

Choi Aj, Park CG, Han SH, Park CB. Nanoemulsion formation and characterization of neutraceutical component with the sonication and self assembly method. NSTI-Nanotech. 2010;1:928-929.

Deng Y, Scherer PE. Adipokines as novel biomarkers and regulators of the metabolic syndrome. Ann N Y Acad Sci. 2010;1212:E1-E19.

Dłużewska E, Stabiecka A, Maszewska M. Effect of oil phase concentration on rheological properties and stability of beverage emulsion. Acta Scient Polonorum Technol Aliment. 2006;5(2):147-156

Dzamko NL, Steinberg GR. AMPK-dependent hormonal regulation of whole-body energy metabolism. Acta Physiol (Oxf). 2009;196(1):115-127.

El-Shazly A, Ateya AM, Wink M. Quinolizidine alkaloid profiles of Lupinus varius orientalis, L. albus albus, L. hertwehii and L. densiflorus. Z Naturforsch. 2001;56(1-2):21-30.

Erbas M. The effects of different debittering methods on the production of lupin bean snack from bitter Lupinus albus 1 . Seeds. J Food Quality. 2010;33(6):742-757.

Galic S, Oakhill JS, Steinberg GR. Adipose tissue as an endocrine organ. Mol Cell Endocrinol. 2010;316(2):129-139.

Gershanik T, Benita S. Self-dispersing lipid formulations for improving oral absorption of lipophilic drugs. Eur J Pharm Biopharm. 2000;50(1):179-188.

Havel PJ. Control of energy homeostasis and insulin action by adipocyte hormones: leptin, acylation stimulating protein, and adiponectin. Curr Opin Lipidol. 2002;13(1):51-59.

Humberstone AJ, Charman WN. Lipid-based vehicles for the oral delivery of poorly water soluble drugs. Adv Drug Delivery Rev. 1997;25(1):103-128.

Jeong YJ, Sohn E-H, Jung Y-H, Yoon W-J, Cho YM, Kim I, et al. Anti-obesity effect of Crinum asiaticum var. japonicum Baker extract in high-fat diet-induced and monogenic obese mice. Biomed Pharmacother. 2016;82:35-43.

Kamba EA, Itodo AU, Ogah E. Utilization of different emulsifying agents in the preparation and stabilization of emulsions. Int J Mat Chem. 2013;3(4):69-74.
Lampart-Szczapa E, Korczak J, Nogala-Kalucka M, Zawirska-Wojtasiak R. Antioxidant properties of lupin seed products. Food Chem. 2003a;83(2):279-285.

Lampart-Szczapa E, Siger A, Trojanowska K, NogalaKalucka M, Malecka M, Pacholek B. Chemical composition and antibacterial activities of lupin seeds extracts. Nahrung. 2003b;47(5):286-290.

Lara-Castro C, Fu Y, Chung BH, Garvey WT. Adiponectin and the metabolic syndrome: mechanisms mediating risk for metabolic and cardiovascular disease. Curr Opin Lipidol. 2007;18(3):263-270.

Lim CT, Kola B, Korbonits M. AMPK as a mediator of hormonal signalling. J Mol Endocrinol. 2010;44(2):87-97.

Mantzoros CS, Magkos F, Brinkoetter M, Sienkiewicz E, Dardeno TA, Kim SY, et al. Leptin in human physiology and pathophysiology. Am J Physiol Endocrinol Metab. 2011;301(4):E567-584.

Medhin DG, Hadhazy, Bakos P, Verzar-Petri G. Hypotensive effects of Lupinus termis and Coriandrum sativum in Anaesthetized rats. A preliminary study. Acta Pharm Hung. 1986;56(2):59-63.

Meier U, Gressner AM. Endocrine regulation of energy metabolism: review of pathobiochemical and clinical chemical aspects of leptin, ghrelin, adiponectin, and resistin. Clin Chem. 2004;50(9):1511-1525.

Nasrallah MP, Ziyadeh FN. Overview of the physiology and pathophysiology of leptin with special emphasis on its role in the kidney. Semin Nephrol. 2013;33(1):54-65.

Obici S, Feng Z, Morgan K, Stein D, Karkanias G, Rossetti L. Central administration of oleic acid inhibits glucose production and food intake. Diabetes. 2002;51(2):271-275.

Oliveira V, Marinho R, Vitorino D, Santos GA, Moraes JC, Dragano N, et al. Diets containing alpha-linolenic (omega3) or oleic (omega9) Fatty acids rescues obese mice from insulin resistance. Endocrinology. 2015;156(11):4033-4046.

PadwalRS, MajumdarSR. Drug treatments for obesity: orlistat, sibutramine, and rimonabant. Lancet. 2007;369(9555):71-77.

Park T, Usher K, Foster K. Description of a healthy lifestyle intervention for people with serious mental illness taking second-generation antipsychotics. Int J Ment Health Nurs. 2011;20(6):428-437.

Raatz SK, Redmon JB, Wimmergren N, Donadio JV, Bibus DM. Enhanced absorption of n-3 fatty acids from emulsified 
compared with encapsulated fish oil. J Am Diet Assoc. 2009;109(6):1076-1081.

Rabe K, Lehrke M, Parhofer KG, Broedl UC. Adipokines and insulin resistance. Mol Med. 2008;14(11-12):741-751.

Savjani KT, Gajjar AK, Savjani JK. Drug solubility: importance and enhancement techniques. ISRN Pharm. 2012;2012:195727.

Sbihi HM, Nehdi IA, Tan CP, Al-Resayes SI. Bitter and sweet lupin (Lupinus albus L.) seeds and seed oils: A comparison study of their compositions and physicochemical properties. Ind Crops Prod. 2013;49:573-579.

Sharma N, Mishra S, Sharma S, Deshpande RD, Sharma RK. Preparation and optimization of nanoemulsions for targeting drug delivery. Int J Drug Develop Res. 2013;5(4):37-48.

Sheweita SA, Newairy AA, Mansour HA, Yousef MI. Effect of some hypoglycemic herbs on the activity of phase I and II drug-metabolizing enzymes in alloxan-induced diabetic rats. Toxicology. 2002;174(2):131-139.
Silva FM, de Almeida JC, Feoli AM. Effect of diet on adiponectin levels in blood. Nutr Rev. 2011;69(10):599-612.

Sipsas S. Lupin products-Concepts and reality. Proceedings of the 12th International Lupin Conference. Lupins for Health and Wealth; 2008.

Stark R, Ashley SE, Andrews ZB. AMPK and the neuroendocrine regulation of appetite and energy expenditure. Mol Cell Endocrinol. 2013;366(2):215-223.

Su J, Ma C, Liu C, Gao C, Nie R, Wang H. Hypolipidemic activity of peony seed oil rich in alpha-linolenic, is mediated through inhibition of lipogenesis and upregulation of fatty acid beta-oxidation. J Food Sci. 2016;81(4):H1001-1009.

Takasawa A, Kato I, Takasawa K, Ishii Y, Yoshida T, Shehata $\mathrm{MH}$, et al. Mutation-, aging-and gene dosage-dependent accumulation of neuroserpin (G392E) in endoplasmic reticula and lysosomes of neurons in transgenic mice. J Biol Chem. 2008;283(51):35606-35613.

Received for publication on $30^{\text {th }}$ September 2017 Accepted for publication on $21^{\text {st }}$ January 2019 